\title{
Supporting information for: Structural Evolution of Solid Phenanthrene at High Pressures
}

\author{
F. Capitani, ${ }^{*}$, @ M. Höppner, ${ }^{\ddagger}$ L. Malavasi, ${ }^{\text {II }}$ C. Marini, ${ }^{\S}$ G.A. Artioli, II M. \\ Hanfland, ${ }^{\S}$ P. Dore, $\|$ L. Boeri, $\stackrel{\perp, \ddagger}{ }$ and P. Postorino $\#$ \\ $\dagger$ †ipartimento di Fisica, Università di Roma Sapienza, P.le Aldo Moro 2, 00185 Roma, Italy \\ $\$$ Max Planck Institute for Solid State Research, Heisenbergstrasse 1, D-70569, Stuttgart, \\ Germany \\ IIDipartimento di Chimica, Università di Pavia, Via Taramelli 16, 27100 Pavia, Italy \\ $\S$ European Synchrotron Radiation Facility, BP 220, 38043 Grenoble Cedex, France \\ \|CNR-SPIN and Dipartimento di Fisica, Università di Roma Sapienza, P.le Aldo Moro 2, 00185 \\ Roma, Italy \\ $\perp$ Institute of Theoretical and Computational Physics, TU Graz, Petersgasse 16, 8010, Graz, \\ Austria \\ \#CNR-IOM and Dipartimento di Fisica, Università di Roma Sapienza, P.le Aldo Moro 2, 00185 \\ Roma, Italy \\ @ Present Address: Synchrotron SOLEIL, L'Orme des Merisiers, F-91192 Gif-sur-Yvette, France \\ E-mail: francesco.capitani@synchrotron-soleil.fr
}

\section{Computational methods}

\section{DFT calculations}

To select a proper functional for the enthalpy calculations we performed a preliminary study of the equation of state of the $\mathrm{P} 21$ ambient pressure phase of phenanthrene $\left(\mathrm{S}_{\mathrm{LP}}\right)$ using different functionals. The results are reported in tables S1 and S2. In general, functionals which include van-der-Waals corrections (vdW-DFT) yield a better agreement with experiment than the standard local density approximation (LDA), which predicts a substantial overbinding.

Of all the functionals in the table, the vdW-DFT functional of Lee et al., ${ }^{\mathrm{S} 7}$ yields overall the best agreement with the experimental data, with lattice constants within $2 \%$ of the experimental values; this is the functional we selected for all further studies. The agreement for the bulk modulus is worse, but this is not uncommon given its ex- tremely small values.

Only after the completion of the present work,

Table S1: Relaxed unit cell parameters at ambient conditions of the $\mathrm{P} 2{ }_{1}$ unit cell for different choices of the DFT exchange-correlation functionals. For all calculations, the local exchange was of Slater-type with $\alpha=2 / 3$. The last two columns indicate the choice of the local correlation functional $\left(\varepsilon_{\mathrm{c}}\right)$, the type of the gradient correction and the van-der-Waals functional $\left(g_{\text {exc }}+v d W\right)$, respectively. Data obtained with the DF2(rPW86) functional employed in the structure search are shown in bold face.

\begin{tabular}{|c|c|c|c|c|c|c|}
\hline & $a(\AA)$ & $b(\AA)$ & $c(\AA)$ & $\beta\left(^{\circ}\right)$ & $\varepsilon_{\mathrm{c}}$ & $\mathrm{vdW}+g_{\mathrm{exc}}$ \\
\hline exp. ${ }^{\text {S1 }}$ & 8.46 & 6.16 & 9.48 & 98.11 & & \\
\hline DFT & 8.08 & 5.81 & 9.06 & 97.05 & $\mathrm{PZ}{ }^{\mathrm{S} 2}$ & - \\
\hline DFT & 8.74 & 6.18 & 9.51 & 98.19 & $\mathrm{PW}^{\mathrm{S} 3}$ & $\mathrm{DF} 1(\mathrm{rPBE})^{\mathrm{S} 4}$ \\
\hline DFT & 8.21 & 5.99 & 9.29 & 96.89 & $\mathrm{PW}^{\mathrm{S} 3}$ & $\mathrm{DF} 1(\mathrm{oB} 88)^{\mathrm{S} 5}$ \\
\hline DFT & 8.19 & 6.00 & 9.32 & 96.68 & $\mathrm{PW}^{\mathrm{S} 3}$ & $\mathrm{DF} 1(\mathrm{oB} 86 \mathrm{~b})^{\mathrm{S} 6}$ \\
\hline DFT & 8.52 & 6.09 & 9.37 & 98.02 & $\mathbf{P W}^{\mathrm{S} 3}$ & DF2(rPW86) ${ }^{\mathrm{S}}$ \\
\hline DFT & 8.26 & 6.00 & 9.33 & 96.94 & $\mathrm{PW}^{\mathrm{S} 3}$ & $\mathrm{DF} 2(\mathrm{rB} 86 \mathrm{~b})^{\mathrm{S} 8}$ \\
\hline
\end{tabular}


Table S2: Equilibrium volumes $V$, compressibilities $B_{0}$ and their derivatives $B_{0}^{\prime}$ fitted with the thirdorder Birch-Murnaghan equation of state ${ }^{\mathrm{S} 9}$ as a function of the exchange and van-der-Waals functional (see Table S1)

\begin{tabular}{|c|c|c|c|c|c|}
\hline & $\varepsilon_{\mathrm{c}}$ & $\mathrm{vdW}+g_{\mathrm{exc}}$ & $V\left(\AA^{3}\right)$ & $B_{0}(\mathrm{GPa})$ & $B_{0}^{\prime}$ \\
\hline exp. & & & $489.0(3)$ & $5.7(6)$ & $9.7(6)$ \\
\hline DFT & $\mathrm{PZ}^{\mathrm{S} 2}$ & - & 423.5 & 12.0 & 9.0 \\
\hline DFT & $\mathrm{PW}^{\mathrm{S} 3}$ & $\mathrm{DF} 1(\mathrm{rPBE})^{\mathrm{S} 4}$ & 508.8 & 7.6 & 7.6 \\
\hline DFT & $\mathrm{PW}^{\mathrm{S3}}$ & $\mathrm{DF} 1(\mathrm{oB} 88)^{\mathrm{S} 5}$ & 453.6 & 11.5 & 8.2 \\
\hline DFT & $\mathrm{PW}^{\mathrm{S3}}$ & $\mathrm{DF} 1(\mathrm{oB} 86 \mathrm{~b})^{\mathrm{S} 6}$ & 454.7 & 10.4 & 8.4 \\
\hline DFT & $\mathbf{P W}^{\text {S3 }}$ & DF2(rPW86) ${ }^{\text {S7 }}$ & 481.6 & 10.2 & 8.3 \\
\hline DFT & $\mathrm{PW}^{\mathrm{S} 3}$ & $\mathrm{DF} 2(\mathrm{rB} 86 \mathrm{~b})^{\mathrm{S} 8}$ & 459.3 & 9.5 & 8.7 \\
\hline
\end{tabular}

our attention was drawn to the vdW-DF1(cx) $)^{\mathrm{S} 10}$ functional. It yields better elastic properties than other vdW-DFT functionals $\left(B_{0}=6.6 \mathrm{GPa}, B_{0}^{\prime}=\right.$ 9.2), but the equilibrium lattice constants are not as good as the ones of vdW-DF(rPW86). ${ }^{\mathrm{S} 7}$ They are $a=8.32 \AA, b=6.04 \AA, c=9.49 \AA$ and $\beta=$ $96.45^{\circ}$.

\section{Evolutionary Algorithm}

To explore the complex state space of solid phenanthrene we employed an evolutionary algorithm to search the structure with the lowest enthalpy for a given pressure, as implemented in the XtalOpt code ${ }^{\mathrm{S} 11-\mathrm{S} 13}$ including optimizations for molecular crystals (E. Zurek, private communications).

For our runs, we used 30 initial structures, a pool of 20 parental structures, 10 cell shape samples, $Z=2 / 3$ molecular movers and 10 volume samples. A run of the evolutionary algorithm was considered converged, when the lowest-enthalpy structure was found to be the same for more than $N_{\min }=4$ generations. Since our approach is not generation-based, we typically end up with more than 10 similar low-enthalpy structures. For each EA run with $Z=2$, we spent on average 1.5 month on 16 nodes with 8 cores being approx. 140.000 CPU hours. For $Z=3$, we needed approx. 400.000 CPU hours. To speed up the search, we used an algorithm specifically designed for molecular solids, which discards all structures which do not respect a given molecular connectivity. This suppresses effectively structures with collapsed or largely distorted molecules. However, some configurations with largely-bent molecules or even fused ones (i.e. with inter- and intramolecular distances between carbon atoms having the same order of magnitude) survive. These structures are referred as "locally amorphous" structures in the text.

To classify the structural differences in our enthalpy vs. volume plots, we use the angle $\vartheta$ between the molecules - see Fig. S1. If the coordinates of individual atoms found in the variable-cell relaxations can be matched to molecules (see below), i.e. if the molecules are not collapsed, the intermolecular angle is defined as:

$$
\vartheta=\frac{2}{Z(Z-1)} \sum_{i, j>i}^{Z-1} \vartheta_{i j}
$$

For $Z \leq 3$, this relation allows an exact mapping onto $[0 \ldots 90]^{\circ}$, and it is used to define the color scale from yellow to red in our state space plots. A structure is considered as collapsed and represented by a green dot if the molecules cannot be matched.

We used the following matching conditions:

(i) planar molecules: check the connectivity of the molecules and do a planar fit. If the distance of an atom from the plane is larger than $0.5 \AA$, the molecule is considered as collapsed.

(ii) lightly bent: if the sum of the distances of the atoms to the respective fitted planes is larger than $Z \times 4 \times 0.5 \AA$, the molecules are considered collapsed.

The first condition ensures that only molecules are matched which are close to be planar. The second condition excludes the cases in which a plane fit was possible, but the molecule itself is too staggered.

\section{Details of low-pressure (LP) and high-pressure (HP) crystal struc- tures}

Typical XRD images of phenanthrene powder in the low pressure and in the high pressure phase 
are shown in Fig.S1(a) and (b) respectively. Twodimensional images are then integrated and converted to one-dimensional XRD patterns which are then analyzed by a Le Bail profile fitting. The crystal structures used to fit these patterns at low and high pressure $-S_{L P}$ and $S_{H P}$ respectively - are shown in Fig. 1 of the main manuscript. In $S_{L P}$ (space group $\mathrm{P} 2{ }_{1}, Z=2$ ) the molecules have a herringbone arrangement within the $a b$ plane; in $\mathrm{S}_{\mathrm{HP}}$ (space group $\mathrm{P} 1, Z=3$ ) the molecules have a parallel arrangement within the $a b$ plane, and the stacking is $\mathrm{AA} \overline{\mathrm{A}}$, i.e. one molecule is flipped by $180^{\circ}$. The results of the Le Bail fit procedure at 2.1 GPa is shown in Figs. S2, where $\mathrm{S}_{\mathrm{LP}}$ is able to well reproduce experimental data. The Le Bail fit of XRD data at $15.5 \mathrm{GPa}$, i.e. when $\mathrm{S}_{\mathrm{HP}}$ is stabilized, is instead shown in Fig. 1 of the main text.

The pressure dependence of the lattice parameters obtained from calculations (lines) and from the fit of XRD data (symbols) is shown in Figs. S3-S4. The agreement is almost perfect for the low-pressure phase, while in the highpressure phase there is a deviation of $2-3 \%$ for the angle $\alpha$ and the in-plane lattice constant $b$. This uncertainty may indicate a perturbation of the long-range order, i.e. locally different aligned molecules deviating from the pattern of the $\mathrm{S}_{\mathrm{HP}}$. We also provide the calculated structures as cifFiles at $0 \mathrm{GPa}$ and $16 \mathrm{GPa}, \mathrm{S}_{\mathrm{LP}}$ and $\mathrm{S}_{\mathrm{HP}}$, respectively.

\section{Other structures}

Fig. S5 displays the two structures $S_{1}$ and $S_{\text {collapsed }}$ which have the lowest enthalpy in our $Z=2$ state space at $P=16 \mathrm{GPa}$ and $P=25 \mathrm{GPa}$, respectively. Both have space group $\mathrm{P} \overline{1}$. In $\mathrm{S}_{1}$ the phenanthrene molecules are arranged parallel to each other $(A \bar{A}$ stacking); in $\mathrm{S}_{\text {collapsed }}$ the molecules are fused so that the original molecular pattern cannot be seen. Some of the double $(\pi)$-bonds are broken to form a fourth bond on the carbon atom. Thus, there are differently coordinated carbon sites ranging from a $\mathrm{sp}^{2}$ bond scenario to a $\mathrm{sp}^{3}$ one. These locally diverse hydrocarbons form $2 \mathrm{D}$ sheets, stacked along the $c$-axis. The concentration of structures of this type increases with pressure in the low-enthalpy region of the phase space.
Note that the structure with the lowest enthalpy per molecule at $P=16 \mathrm{GPa}$ is $\mathrm{S}_{1}$ and not $\mathrm{S}_{\mathrm{HP}}$, but neither $S_{1}$, nor any other structure with $Z=2$ in the vicinity of the global minimum could fit the experimental XRD spectra, while several structures with $Z=3$ fit the experimental data quite well. The enthalpy difference is of the order of $70 \mathrm{meV}$ per molecule, i.e. within the uncertainty due to zero-point motion and anharmonic corrections. Another possible explanation why the $S_{1}$ is not observed in the experiment might be a much larger energy barrier associated to the higher number of molecular flips in the $Z=2$ structure.

\section{References}

(S1) Trotter, J. Acta Crystallogr. 1963, 16, 605608.

(S2) Perdew, J. P.; Zunger, A. Phys. Rev. B 1981, 23, 5048-5079.

(S3) Perdew, J. P.; Wang, Y. Phys. Rev. B 1992, 45, 13244-13249.

(S4) Zhang, Y.; Yang, W. Phys. Rev. Lett. 1998, 80, 890-890.

(S5) Klimě̌, J.; Bowler, D. R.; Michaelides, A. J. Phys.: Condens. Matter 2010, 22, 022201.

(S6) Klimeš, J. c. v.; Bowler, D. R.; Michaelides, A. Phys. Rev. B 2011, 83, 195131.

(S7) Murray, a. D.; Lee, K.; Langreth, D. C. Journal of Chemical Theory and Computation 2009, 5, 2754-2762.

(S8) Hamada, I. Phys. Rev. B 2014, 89, 121103.

(S9) Birch, F. Phys. Rev. 1947, 71, 809-824.

(S10) Berland, K.; Hyldgaard, P. Phys. Rev. B 2014, 89, 035412.

(S11) Lonie, D. C.; Zurek, E. Comput. Phys. Commun. 2011, 182, 372 - 387.

(S12) Lonie, D. C.; Zurek, E. Comput. Phys. Commun. 2011, 182, 2305 - 2306. 
(S13) Lonie, D. C.; Zurek, E. Comput. Phys. Commun. 2012, 183, 690 - 697. 

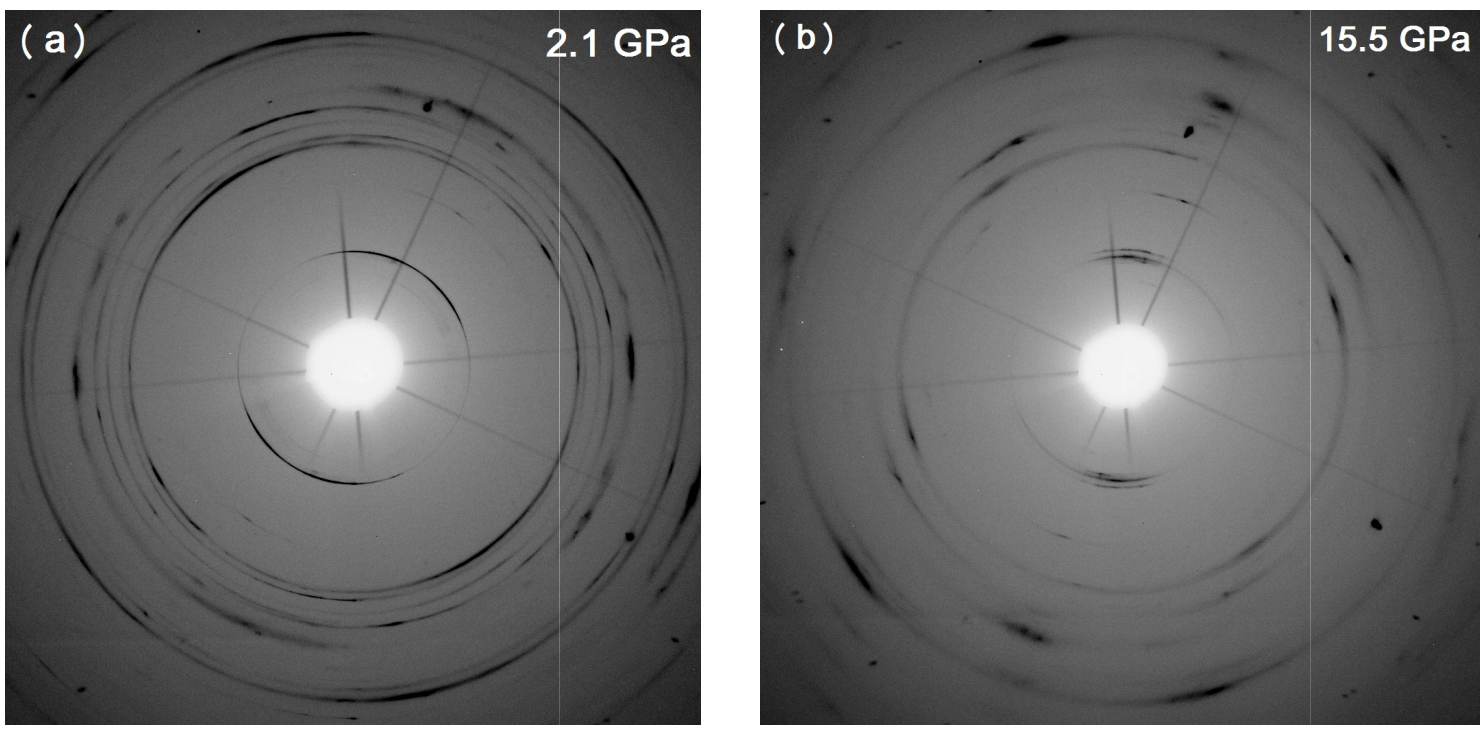

Figure S1: XRD images of phenanthrene powder at 2.1 GPa (a) and $15.5 \mathrm{GPa}(\mathrm{b})$. The images are limited to $2 \theta=8^{\circ} . \lambda=0.4127 \AA$

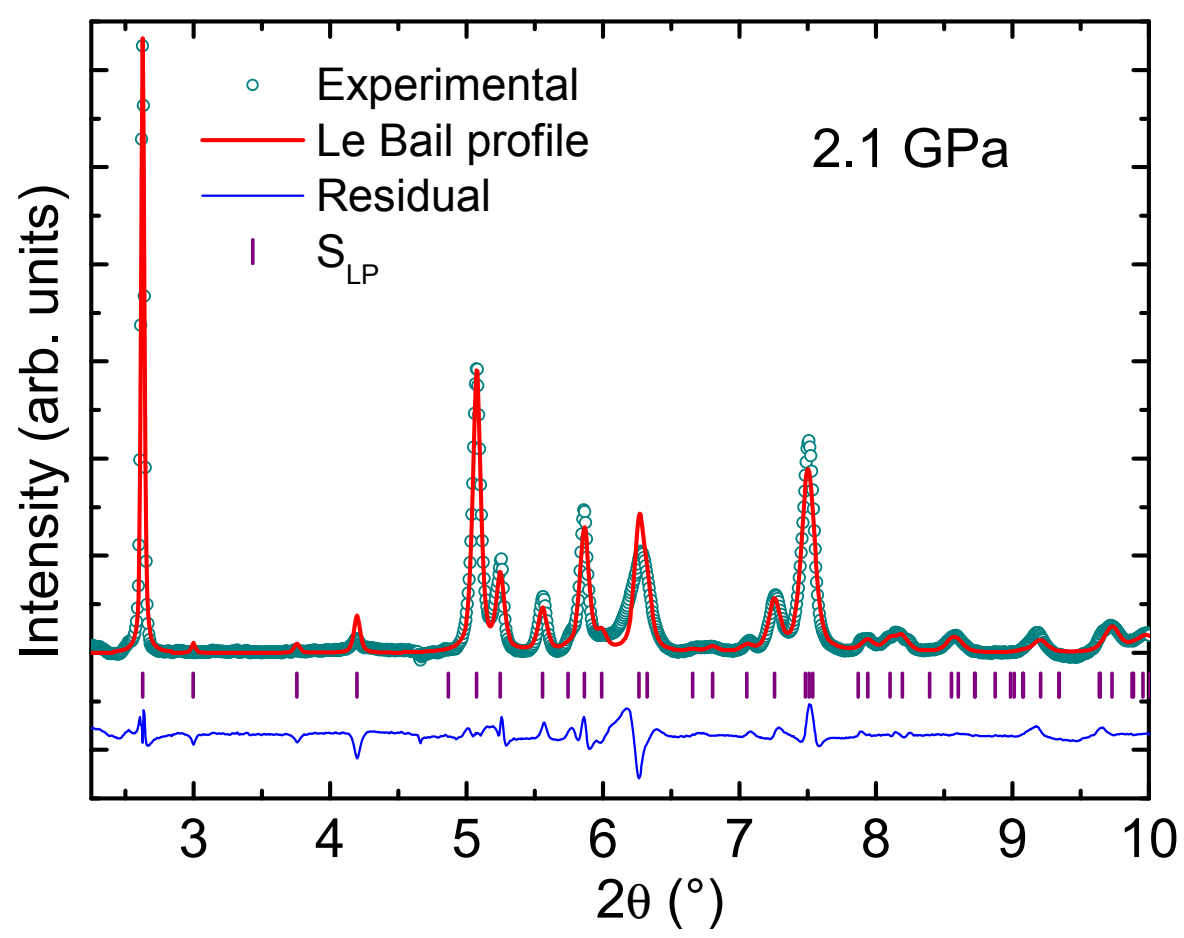

Figure S2: Le Bail profile fitting of the diffraction pattern at $2.1 \mathrm{GPa}$ with the low pressure P $2_{1}$ phase $\mathrm{S}_{\mathrm{LP}}$. 

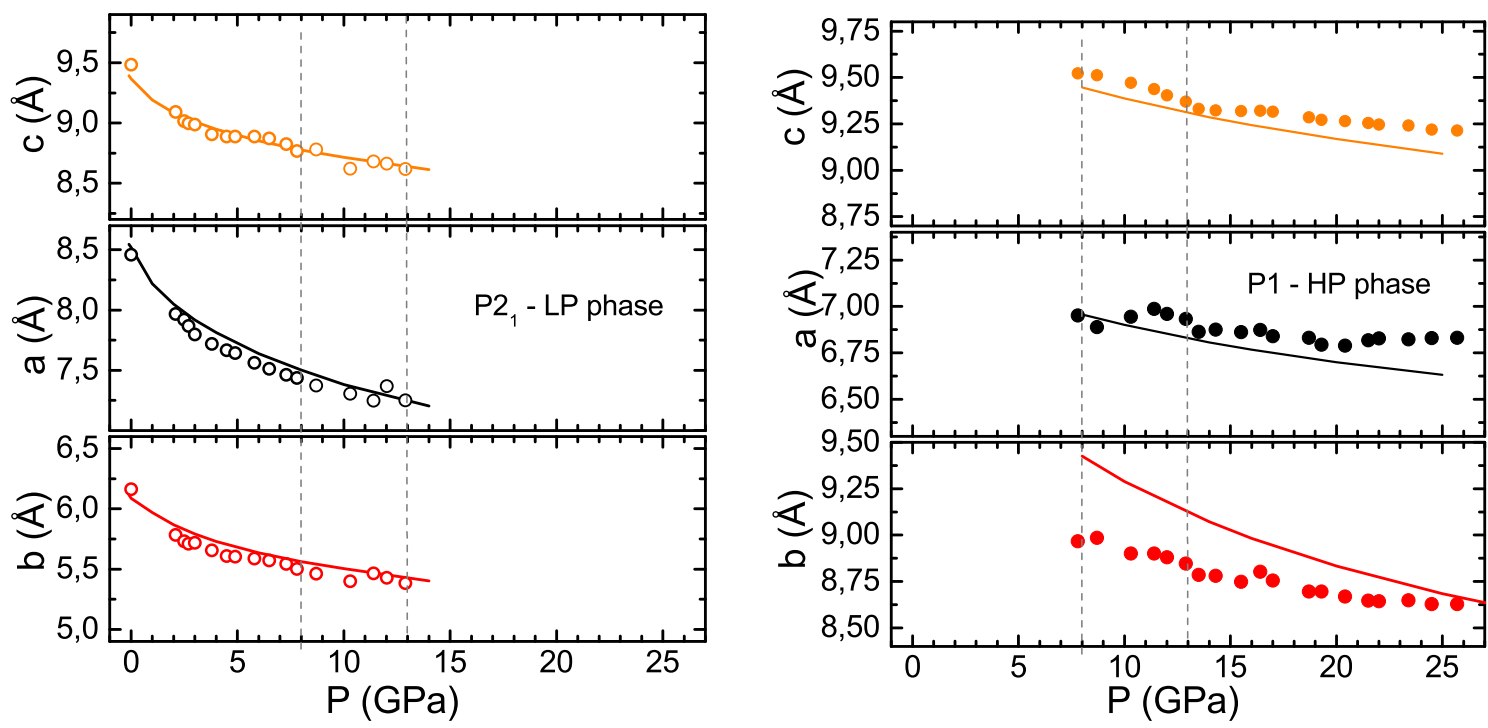

Figure S3: Pressure dependence of the lattice parameters of the LP and HP phase, left and right panel, respectively. Symbols represent data obtained from the Le Bail fit of the measured patterns, whereas solid lines are those obtained from calculations.
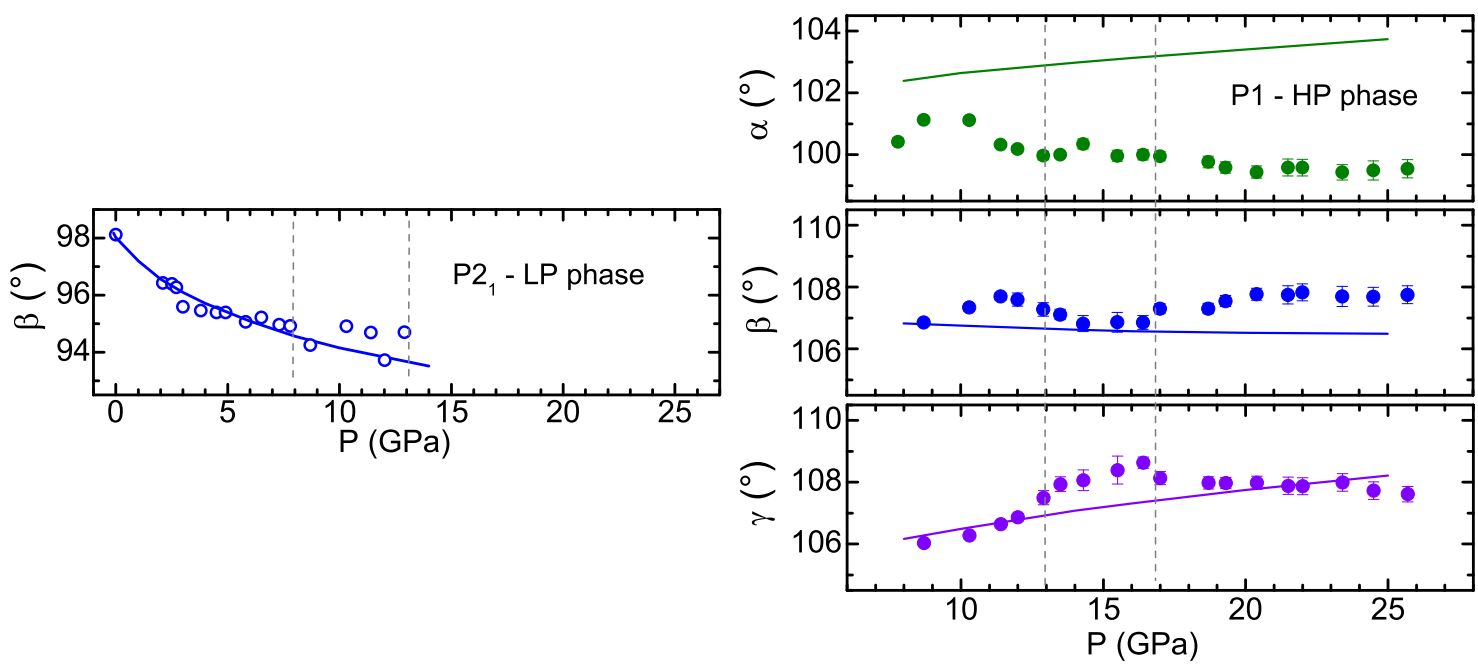

Figure S4: Pressure dependence of the unit cell angles of the LP and HP phase, left and right panels respectively. Symbols represent data obtained from the Le Bail fit of the measured patterns, whereas solid lines are those obtained from calculations. 

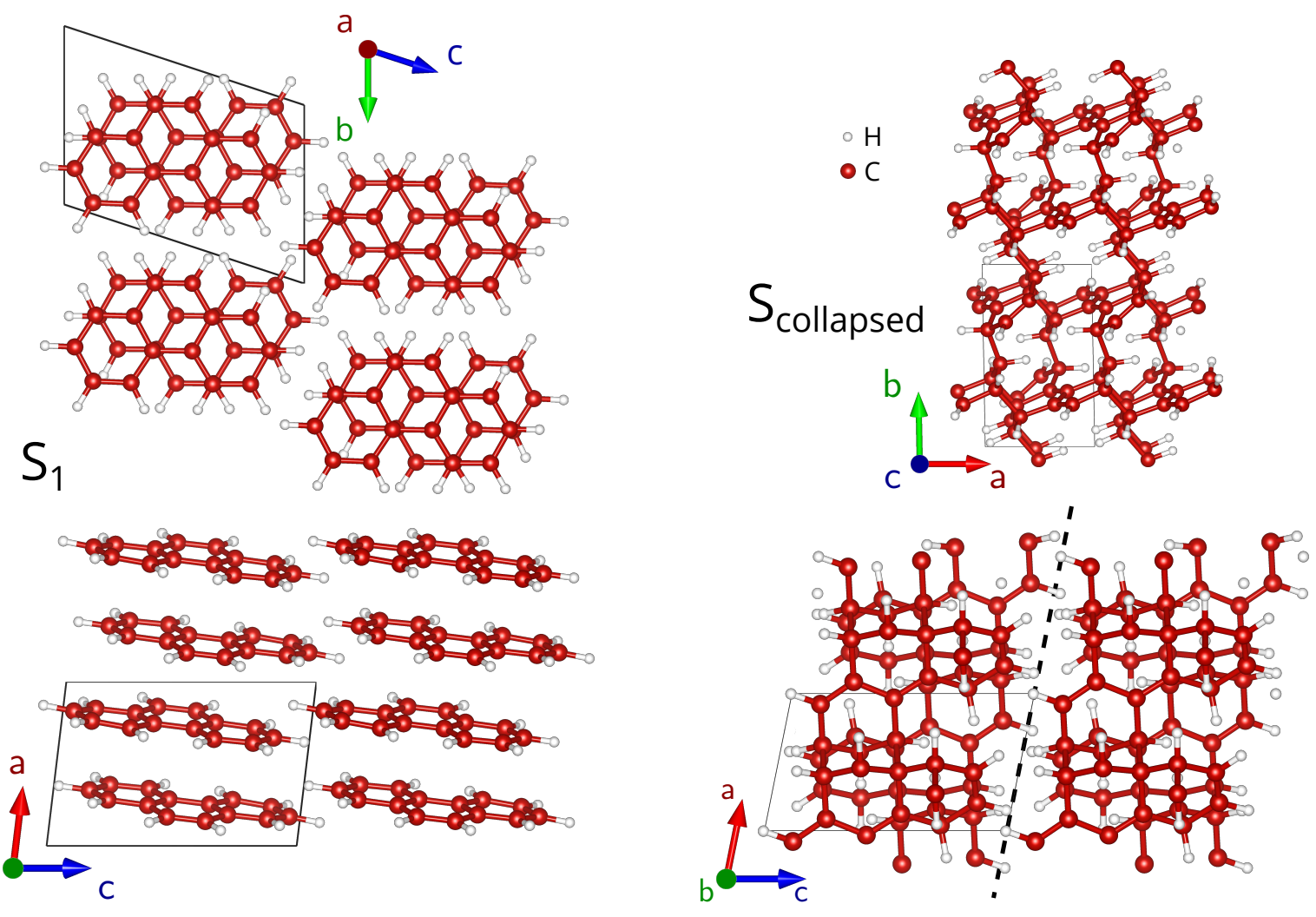

Figure S5: Overview of the lowest-enthalpy crystal structures for $Z=2$ at $16\left(\mathrm{~S}_{1}\right)$ and $25 \mathrm{GPa}\left(\mathrm{S}_{\text {collapsed }}\right)$; $S_{1}$ is similar to $S_{H P}$, however each second molecule is flipped; bottom right $-S_{\text {collapsed }}$ molecular-collapsed ("locally amorphous") structure. The unit cell is drawn with black lines, and the separation of the quasi 2D layers is indicated by a broken line. 